\title{
The use of GPS buoys in the determination of oceanic variables
}

\author{
Estel Cardellach, Dirk Behrend, Giulio Ruffini, and Antonio Rius \\ Institut d'Estudis Espacials de Catalunya (IEEC), CSIC Research Unit, Barcelona, Spain
}

(Received January 11, 2000; Revised June 29, 2000; Accepted June 30, 2000)

\begin{abstract}
GPS observables taken from light-weight GPS buoys more than $80 \mathrm{~km}$ from the GPS reference stations have been analysed using different noise models in the parameter estimation process. The time series solution of the GPS buoy positioning have been used to extract values of oceanic variables (sea level, tides and waves) and tropospheric information. These variables are compared with data from models and with measurements from a meteorological buoy.
\end{abstract}

\section{Introduction}

In preparation of the calibration of ESA's ENVISAT satellite radar altimeter two training GPS buoy field campaigns have been performed in the Mediterranean Sea off the coast of Catalonia in the frame of the so-called GPS Radar Altimeter Calibration (GRAC) project. Previous altimeter calibration campaigns have employed GPS buoys with distances to GPS reference sites not exceeding $20 \mathrm{~km}$ (Christensen et al., 1994; Born et al., 1994; Key et al., 1999; Zilkoski et al., 1999). In the GRAC experiments, the nearest reference station was at $80 \mathrm{~km}$, which, in principle, makes unreliable the use of the tropospheric solution from the reference site. Apart from using the results in the actual calibration process, the GPS data may also be applied to other fields provided that parameter estimates of the GPS buoy are accurate enough. As possible applications we mention here: ground truth in GPS reflection projects, validation of ocean tide models, validation of ocean wave models, derivation of tropospheric information for offshore areas.

Here we focus on the processing of GPS data from lightweight buoys. The position solution is used to determine oceanic variables which finally are compared with ocean tide and meteorological models in the study area of the Western Mediterranean Sea. Furthermore, we conducted comparisons with wave and troposphere information sources. To this end the GPS data collected with antennas on the floating buoys and on land-based reference stations have been processed using the GIPSY/OASIS-II software package developed at JPL (Webb and Zumberge, 1997). For the comparison of GPS buoy data with independently derived variables, we used the CSR-3.0 tidal model (Eanes and Bettadpur, 1995), the tropospheric models included in the operational data of ERS altimeter (ERS-OPR) (see RA/ATSR, 1996), and the meteorological data measured by a meteo-buoy during the campaign.

Copy right (C) The Society of Geomagnetism and Earth, Planetary and Space Sciences (SGEPSS); The Seismological Society of Japan; The Volcanological Society of Japan; The Geodetic Society of Japan; The Japanese Society for Planetary Sciences.

\section{Field Campaigns and Employed Instrumenta- tion}

The first campaign (GRAC99a) was performed under pass No. 773 of ERS-2, at approximately 20 nautical miles off the Catalonian Coast on May 16th, 1999, under severe weather conditions. The second trial (GRAC99b) took place June 16th, 17th, 19th, 20th, 1999, with calm seas following tracks of the ERS-1 (active for this experiment), ERS-2 and TOPEX/POSEIDON satellite radar altimeters in the area between the Catalonian Coast and the Balearic Islands (see Fig. 1). During the five hours around a satellite over-pass, the GPS antennas continuously took observations at a frequency of $1 \mathrm{~Hz}$. In order to counteract the drifting away of the buoy due to ocean surface currents, the buoy was pulled back (at speeds of up to 2 knots) to its desired location by the vessel at certain times. In this way the buoy was maintained within a region of about $1 \mathrm{~km}$ radius of the altimeter ground track with the buoys' trajectory alternating between phases of free floating and power-driven pulling (see inset of Fig. 1). This procedure is a trade-off between instrumental, hydrographic and calibration issues: Instrumental issues comprise the securing of GPS buoy phase continuity over several hours, the accuracy of the predicted altimeter satellite orbit, and the accuracy of the real-time positioning. Hydrographic issues relate to the difficulty to predict the ship's heading in the free floating phase due to the ocean currents. However, the free floating phase is more reliable for altimeter calibration purposes, since there are no additional dynamic effects.

In the GRAC99b experiment, three toroidal buoys were deployed and attached to each other in such a way that a $2.8 \mathrm{~m}$ edge length equilateral triangle was formed (see Fig. 2). The distance between buoys was chosen for minimizing the multi-path effect (Elósegui et al., 1995), whereas the joined structure facilitates the single buoys to follow the sea surface movements. While two of the buoys were equipped with a GPS antenna, the third one carried a replacement of equal weight. The antennas were Trimble and Micropulse chokering antennas, each of them connected to a separate Trimble 4000 receiver through 50 meters of coaxial cable. This setup was chosen in order to facilitate a check of the solution as 


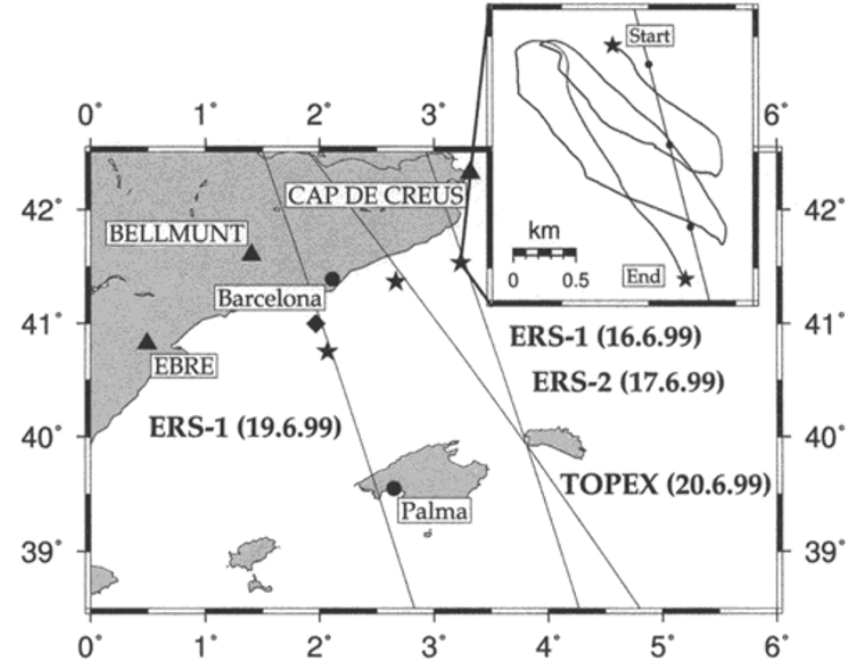

Fig. 1. GRAC99 campaigns: GPS buoy sites marked by a diamond (GRAC99a) and stars (GRAC99b). Inset: GRAC99b, June 17th, 1999: Buoy trajectory and ERS-2 predicted over-flight. Free floating phase for south-east tracks, pulling phase for north-west tracks.

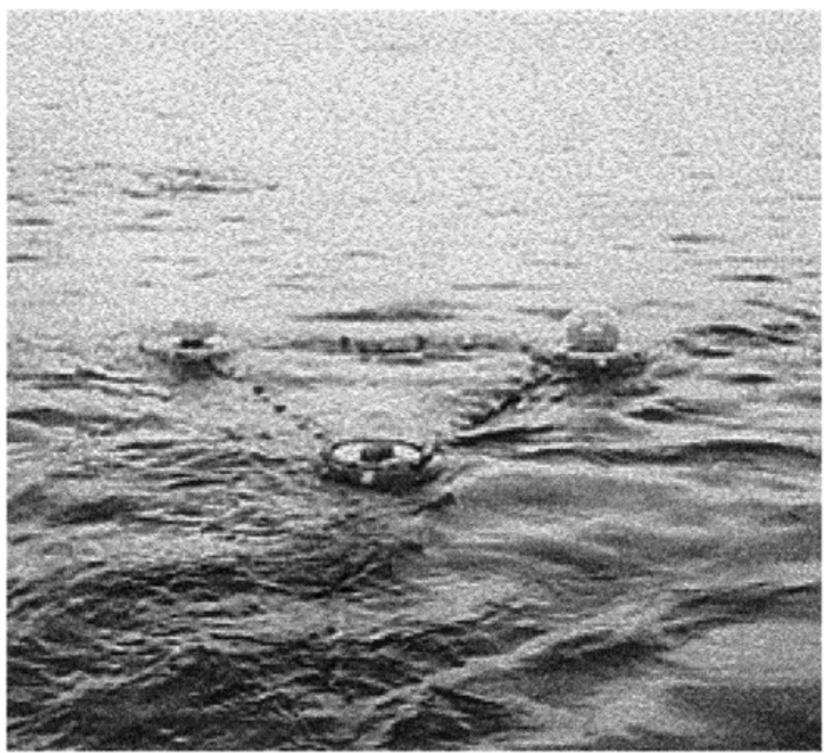

Fig. 2. 3-buoy system deployed in the GRAC99b campaign.

well as to be able to fill possible data gaps.

A coastal monitoring buoy (AANDERAA CMB 3280) was deployed close to the GPS buoy observation area (99.06.16, 99.06.17 and 99.06.20), giving wave, wind and currents observables at a sampling rate of 10 minutes. The resolution of the SWH measurement is $0.01 \mathrm{~m}$, but the accuracy is the 10 per cent of the SWH estimate.

\section{Data Processing}

The two-frequency GPS buoy data have been analyzed with data of the GPS ground stations BELLMUNT, EBRE and CAP de CREUS (see Fig. 1). These stations are part of the permanent GPS network of the Institut Cartogràfic de Catalunya (ICC). Due to the random vertical movements of the buoy, it is difficult to distinguish between tropospheric signal delays, clock errors and the vertical position component of the buoy. In addition, the randomness of the buoys' position imposes another limitation: for estimating the GPS carrier phase biases a sufficiently long observation time is needed (more than one hour).

We have set up a 3-step data analysis strategy based on GIPSY/OASIS-II. In the first step the reference ground stations are positioned using precise GPS orbits and clocks from JPL as well as information on eclipsed satellites and polar motion. The estimated position and tropospheric parameters for the reference stations are then used to calibrate the GPS satellite clocks at the desired frequency (step 2). This is achieved by means of an estimation process where almost all parameters (ground station position and troposphere) are considered known and fixed to the values of the solution of step 1. Hence, the unknowns reduce to the satellite clock offsets at a high sampling rate and the initial phase biases of the data arcs. We used sampling intervals of 1 and 15 seconds: the first one to extract the high frequency part of the sea wave spectrum and the second one to allow longer solutions that are more reliable in the vertical component.

With precise satellite clock information at the desired sampling rate, the "Precise Point Positioning" technique (PPP) (Zumberge et al., 1997) can be applied to the buoy data. The PPP is a strategy, developed at JPL, which uses precise orbits and clocks in order to determine precise coordinates station by station. At the end of each process we apply the carrier phase ambiguity resolution technique (Blewitt, 1989) to set double differenced phase biases to best integer values.

Several noise models have been applied to the estimable parameters in the buoy positioning (step 3), whose effects on the solution are presented in next section. The coordinate noise of the buoy is modelled as a random walk process. The GPS buoy receiver clock offsets were treated either as stochastic white noise or as tight random walk processes. The tropospheric delay has been estimated using different approaches: as an offset with respect to the time-dependent solution of a reference station, as a batched constant bias (approximately 1 solution per hour), and as a random walk process with 1 hour intervals between updates. The use of both antennas to constrain the tropospheric solution has also been tested.

\section{Preliminary Results}

Short period wave information can be extracted from the $1 \mathrm{~Hz}$ solution. Figure 3 shows a detailed sequence ( $20 \mathrm{sec}-$ onds) of the 3-dimensional time series derived from data of the GRAC99a campaign. In the data analysis a batched solution was employed treating the receiver clock errors as a tight random walk process and the tropospheric delay as a constant bias with respect to the time-dependent tropospheric solution of station BELLMUNT ("compartial troposphere"). For large distances (e.g. $80 \mathrm{~km}$ ) between the reference site (ground station) and the buoy, the tropospheric behaviour at the ground station differs too much from the behaviour at the buoy so that a link by a constant offset is not feasible. Thus, the compartial approach results in insufficient repeatabilities of the vertical and tropospheric parameter estimates, because the two parameters are highly correlated and strongly influ- 


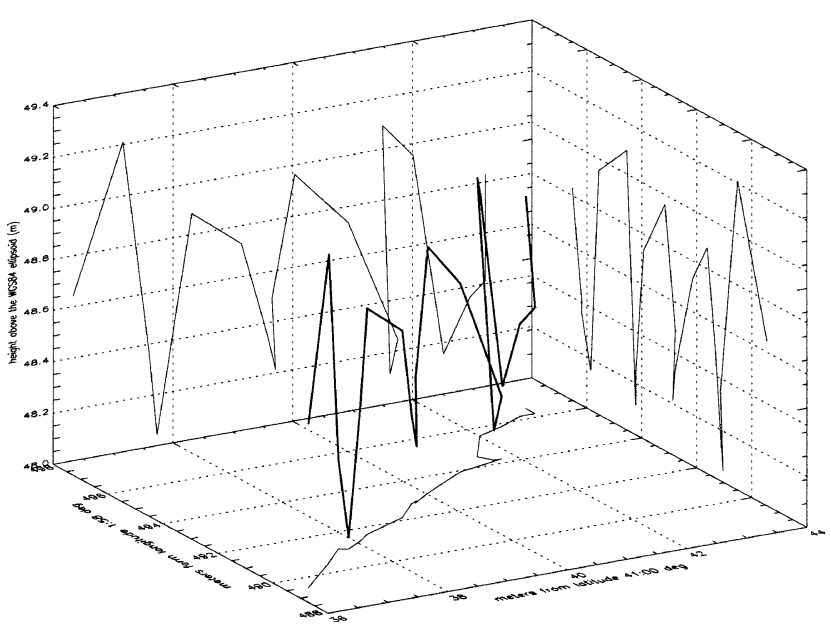

Fig. 3. 20 seconds of the 3-dimensional time series solution from data gathered in GRAC99a campaign. The thin lines are the projections to the latitude-longitude, latitude-height and longitude-height planes. Longitude and latitude are in meters from $1^{\circ} 58^{\prime}$ and $41^{\circ} 00^{\prime}$, respectively.

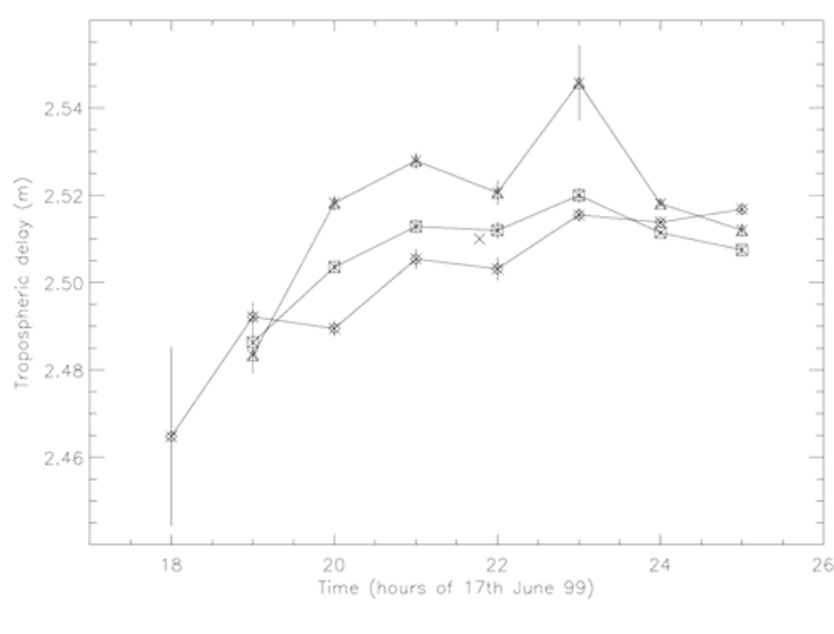

Fig. 4. GRAC99b (99.06.17) total tropospheric zenith delay estimate for independent runs (diamond and triangles) and common, shared tropospheric parameters solution (squares). The ERS-2 correction, computed through numerical models, is the single cross. The radiometer correction was not available. The effect of using the common troposphere as a constraint is here displayed. enced by the reference station estimates. Accordingly, the "compartial approach" is inapt for altimeter calibration purposes at these distances.

Something similar happens when the buoys' clock errors are treated as a tight random walk and the tropospheric parameters as batched constants. In this case, clock jumps are truncated in the solutions and, at times, the observation length is too short to properly estimate the initial integer Doppler phase cycles, introducing an additional error in the form of offsets in the sea level measurements at each clock jump. But still, this approach is useful for determining the roughness of the sea surface at the moment of the altimeter observation: the standard deviation for an extract of 15 minutes of the time series around the ERS-2 over-pass on June 17th, 1999 amounts to $\sigma_{z}=6.4 \pm 1.8 \mathrm{~cm}$. The significant wave height (SWH) is related to $\sigma_{z}$ by $\mathrm{SWH}=H_{1 / 3}=4 \cdot \sigma_{z}$ (e.g. Stewart, 1985). This yields $\mathrm{SWH}=26 \pm 7 \mathrm{~cm}$. While the agreement with the ERS value $\mathrm{SWH}_{\mathrm{ERS}}=11 \pm 1 \mathrm{~cm}(\mathrm{ESA}, 1999)$ is low, the agreement with the meteo-buoy placed about $6 \mathrm{~km}$ from the GPS buoys site with $\mathrm{SWH}=20 \pm 2 \mathrm{~cm}$ is quite good. The discrepancies with the altimetric measurement might be caused by the fact that it treats the space-time coverage in a different way.

In order to show tropospheric delays derived for the GPS buoy we present results from the GRAC99b campaign. The tropospheric delay was modelled as a random walk process with 1 hour of update intervals and the receiver clock offsets were handled as white noise. Figure 4 shows independent tropospheric estimates determined for each buoy separately as well as forced common tropospheric delays obtained by processing both antennas simultaneously. In the instant of the altimeter over-pass (1999.06.17) the tropospheric GPS delay estimate and its formal error amounts to $2.512 \pm 0.002 \mathrm{~m}$. This value compares well with $2.51 \mathrm{~m}$, the data given in the ERS-OPR CDROM (ESA, 1999), which was estimated from meteorological models.

The sea level has been analyzed for the days 1999.06.17 and 1999.06.19 of the GRAC99b campaign. Due to higher reliability of the common tropospheric solution for both buoys, we present here the solutions from this procedure. The buoys' receiver clock error has been modeled as white noise to avoid truncated solutions. A first check of our solution was the comparison with a tidal model in order to evaluate the time evolution performance. We used the CSR3.0 global tidal model (Eanes and Bettadpur, 1995). The signal in the smoothed height is stronger than the tide model estimate (see Figs. 5 and 6), with a period of about 2 hours and an amplitude of about $20 \mathrm{~cm}$. This signal is slightly correlated with the buoys' trajectory, which at the same time is dominated by two phases: the free floating phase (downstream) and the pulling phase (upstream). Possible reasons for this are the wash of the vessel, the rotation of the antennas at turnings in the trajectory (the amplitude $\Delta h$ is related to the wavelength of L1), or interacting forces between the two buoys through the rigid bar.

15 minutes of data about the ERS-2 over-pass from 1999.06.17 experiment have been averaged to compare with the OPR-2 data. The independent buoy average gives: MICR : 48.112 $\pm 0.078, T R I M: 48.114 \pm 0.072$, which include the antenna phase offset effect. Thus, the 2-antenna averaged sea-level becomes $48.113 \pm 0.053$. The $1 \mathrm{~Hz}$ operational data from ERS passing close to the buoy, at $\mathrm{N}$ $41: 31: 54.4656$, E 3:15:20.268 is $48.226 \pm 0.032$, where the satellite-range measurement has been corrected with dry, wet, ionospheric and sea state bias corrections. The error was taken as the standard deviation of the $20 \mathrm{~Hz}$ individual ERS estimates. The bias obtained by means of this simple comparison is, thus, $11 \mathrm{~cm}$ ERS measurement above the GPS buoy solution. According to Stum et al., the relative bias of the sea surface height (SSH) between T/P and ERS-2 is 2 to $5 \mathrm{~cm}$ (ERS-2 higher), which, in addition to the absolute bias of the T/P, $1 \mathrm{~cm}$ above the real surface, amounts to +3 to $+6 \mathrm{~cm}$ of ERS- 2 absolute bias. This is a smaller value than our $11 \mathrm{~cm}$ estimate, corresponding to a single experiment and compared to a simple OPR data. Note that this disagree- 


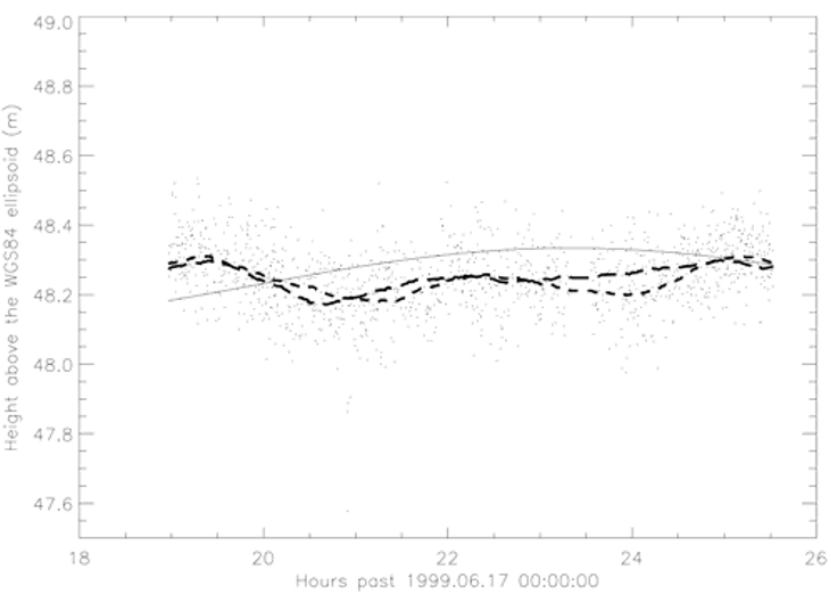

Fig. 5. GRAC99b (99.06.17) SSH GPS solution. Trimble antenna smoothed estimates in dashed, Micro-pulse antenna estimates in long dashed. CSR 3.0 tide estimate (biased for mapping purposes) is the solid thin line.

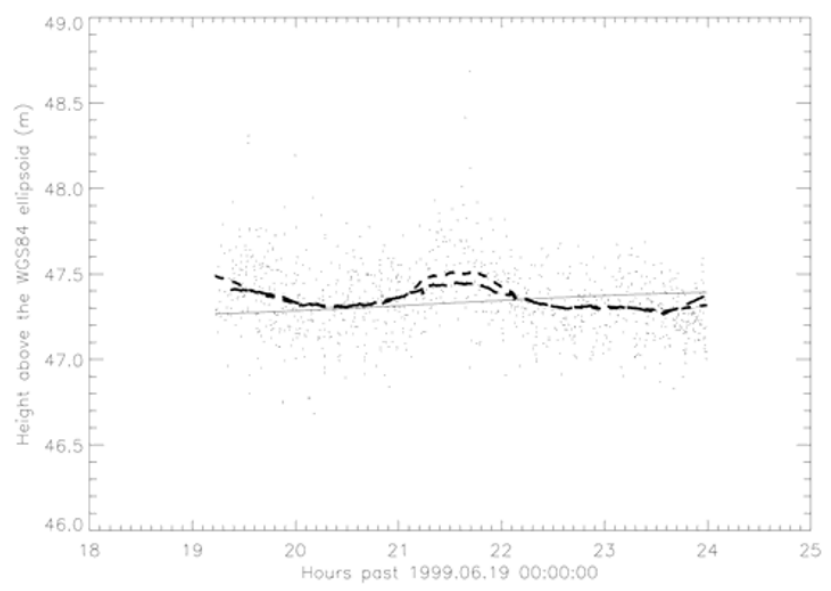

Fig. 6. GRAC99b (99.06.19) SSH GPS solution. Trimble antenna smoothed estimates in dashed line, Micro-pulse antenna estimates in long dashed. CSR 3.0 tide estimate (biased for mapping purposes) is the solid thin line.

ment is within the amplitude of the fluctuant signal, the real accuracy limit in this particular experiment.

\section{Conclusions}

GPS data taken at a high sampling rate from light buoys more than $80 \mathrm{~km}$ from the reference station were analyzed using a 3-step strategy, based on GIPSY/OASIS-II. From $1 \mathrm{~Hz}$ solutions an estimate for the SWH in form of the standard deviation of the time series was derived. The obtained result does not fit to the ERS SWH estimate, but appears reasonably similar to the meteo-buoy measured mean wave height. Initial comparisons indicate that—even at $80 \mathrm{~km}$ off the GPS reference site-the estimation of tropospheric delays appears to be possible with a precision of better than 0.5 $\mathrm{cm}$. But as our comparison reduces to a single point, a more robust check is needed.

The sea level GPS solution appears quite different from the one modelled by a numerical tide model, showing an unexpected signal of $20 \mathrm{~cm}$ of amplitude which is correlated to the vessel trajectory and its power phases. This still is an open issue and needs further investigation in order to avoid corrupted GPS observations in the next campaigns. A single comparison with the ERS-2 sea level estimate gives a bias of $11 \mathrm{~cm}$ between the two sources. This exceeds the expected offset range $(3$ to $6 \mathrm{~cm}$ ) but is inside the amplitude of the low frequency noise $(20 \mathrm{~cm})$, which might be considered the limit of the accuracy in this particular experiment.

Acknowledgments. This study is part of the GRAC project (GPS Radar Altimeter Calibration), funded by the Spanish National Program on Marine Science and Technology and FEDER fond (2FD870588).

\section{References}

Blewitt, G., Carrier phase ambiguity resolution for the global positioning system applied to geodetic baselines up to $2000 \mathrm{~km}$, J. Geophys. Res. 94(B8), 10,187-10,203, 1989.

Born, G. H., M. E. Parke, P. Axelrad, K. L. Gold, J. Johnson, K. W. Key, and D. G. Kubitschek, Calibration of the TOPEX altimeter using a GPS buoy, J. Geophys. Res., 99(C12), 24,517-24,526, 1994.

Christensen, E. J, B. J. Haines, S. J. Keihm, C. S. Morris, R. A. Norman, G. H. Purcell, B. G. Williams, B. D. Wilson, G. H. Born, M. E. Parke, S K. Gill, C. K. Shum, B. D. Tapley, R. Kolenkiewicz, and R. S. Nerem, Calibration of TOPEX/POSEIDON at platform harvest, J. Geophys. Res., 99(C12), 24,465-24,485, 1994.

Eanes, R. and S. Bettadpur, The CSR 3.0 global ocean tide model, Center for Space Research, Technical Memorandum, CSR-TM-95-06, December 1995.

Elósegui, P., J. L. Davis, R. T. Jalkehag, J. M. Johansson, A. E. Niell, and I. I. Shapiro, Geodesy using the Global Positioning System: The effects of signal scattering on estimates of site position, J. Geophys. Res., 100(B7), 9921-9934, 1995.

ESA, ERS-2 Altimeter Ocean Products, F2A0043_1_IC, Version 6, ESAIFREMER 1999.

Key, K. W., G. M. Born, K. D. Leaman, and P. Vertes, A new GPS data processing algorithm for the positioning of oceanographic experiments, Journal of Atmos. Oceanic Technol., 16(8), 1127-1137, 1999.

RA/ATSR products-User Manual, Altimeter and Microwave Radiometer ERS products, C2-MUT-A-01-IF 2.2, 1996.

Stewart, R. H., Methods of Satellite Oceanography, University of California Press, U.S.A., 1985.

Stum, J., F. Ogor, and J. Dorandeu, A comparison study of TOPEX/ POSEIDON, ERS-1 and ERS-2 altimeter and radiometer data, Report of task 4 of IFREMER contract n.99/2.210 765, CLS/DOS/NT/00.233, December 1999

Webb, F. H. and J. F. Zumberge, An Introduction to GIPSY/OASIS-II, JPL D-11088, June 1997.

Zilkoski, D. B., J. D. D'onofrio, R. J. Fury, and C. L. Smith, Centimeterlevel positioning of a US Coast Guard buoy tender, GPS Solutions, $\mathbf{3}(2)$, 53-65, 1999.

Zumberge, J. F., M. B. Heflin, D. C. Jefferson, M. M. Watkins, and F. H. Webb, Precise point positioning for the efficient and robust analysis of GPS data from large networks, J. Geophys. Res., 102(B3), 5005-5017, 1997.

E. Cardellach (e-mail: estel@ieec.fcr.es), D. Behrend, G. Ruffini, and A. Rius 